Supporting Information for

\title{
A Specularity Coefficient Model and Its Application to Dense Particulate Flow Simulations
}

\author{
Yunhua Zhao, Tianqiang Ding, Liangyou Zhu, and Yingjie Zhong* \\ Institute of Energy and Power Engineering, Zhejiang University of \\ Technology, Hangzhou 310014, China
}

Corresponding author

*E-mail: zhong_yingjie@ zjut.edu.cn

Tel./Fax: +86-571-88320650. 
This supporting information contains the following tables:

Table S1. Summary of the governing equations in the present two-fluid model

Table S2. Frictional stress model for particle phase

Table S3. Effective restitution coefficient model 
Table S1. Summary of the governing equations in the present two-fluid model

Continuity equations

$$
\begin{aligned}
& \frac{\partial}{\partial t}\left(\varepsilon_{g} \rho_{g}\right)+\nabla \cdot\left(\varepsilon_{g} \rho_{g} \mathbf{u}_{g}\right)=0 \\
& \frac{\partial}{\partial t}\left(\varepsilon_{p} \rho_{p}\right)+\nabla \cdot\left(\varepsilon_{p} \rho_{p} \mathbf{u}_{p}\right)=0
\end{aligned}
$$

Momentum equations

$$
\begin{aligned}
& \frac{\partial}{\partial t}\left(\varepsilon_{g} \rho_{g} \mathbf{u}_{g}\right)+\nabla \cdot\left(\varepsilon_{g} \rho_{g} \mathbf{u}_{g} \mathbf{u}_{g}\right)=\nabla \cdot\left(\varepsilon_{g} \boldsymbol{\tau}_{g}\right)-\varepsilon_{g} \nabla p_{g}-K\left(\mathbf{u}_{g}-\mathbf{u}_{p}\right)+\varepsilon_{g} \rho_{g} \mathbf{g} \\
& \frac{\partial}{\partial t}\left(\varepsilon_{p} \rho_{p} \mathbf{u}_{p}\right)+\nabla \cdot\left(\varepsilon_{p} \rho_{p} \mathbf{u}_{p} \mathbf{u}_{p}\right)=\nabla \cdot\left(\boldsymbol{\tau}_{p}+\boldsymbol{\tau}_{f}\right)-\varepsilon_{p} \nabla p_{g}+K\left(\mathbf{u}_{g}-\mathbf{u}_{p}\right)+\varepsilon_{p} \rho_{p} \mathbf{g}
\end{aligned}
$$

Gas stress tensor

$$
\boldsymbol{\tau}_{g}=2 \mu_{g} \mathbf{S}_{g}, \text { where } \mathbf{S}_{g}=\frac{1}{2}\left(\nabla \mathbf{u}_{g}+\nabla \mathbf{u}_{g}^{T}\right)-\frac{1}{3}\left(\nabla \cdot \mathbf{u}_{g}\right) \mathbf{I}
$$

Particle phase kinetic-collisional stress tensor

$$
\begin{aligned}
\boldsymbol{\tau}_{p}= & {\left[-p_{p}+\xi_{p}\left(\nabla \cdot \mathbf{u}_{p}\right)\right] \mathbf{I}+2 \mu_{p} \mathbf{S}_{p}, \text { where } \mathbf{S}_{p}=\frac{1}{2}\left(\nabla \mathbf{u}_{p}+\nabla \mathbf{u}_{p}^{T}\right)-\frac{1}{3}\left(\nabla \cdot \mathbf{u}_{p}\right) \mathbf{I} } \\
p_{p} & =\left[1+2(1+e) \varepsilon_{p} g_{0}\right] \varepsilon_{p} \rho_{p} \Theta \\
\mu_{p} & =\frac{4}{5}(1+e) \rho_{p} \varepsilon_{p}^{2} g_{0} d_{p} \sqrt{\frac{\Theta}{\pi}}+\varepsilon_{p} \rho_{p} \Theta \frac{\left[1+\frac{4}{5}(1+e) \varepsilon_{p} g_{0}\right]\left[1+\frac{2}{5}(1+e)(3 e-1) \varepsilon_{p} g_{0}\right]}{\frac{2 K}{\varepsilon_{p} \rho_{p}}+\frac{24(1+e)(3-e) \varepsilon_{p} g_{0}}{5 d_{p}} \sqrt{\frac{\Theta}{\pi}}} \\
\xi_{p} & =\frac{4}{3}(1+e) \rho_{p} \varepsilon_{p}^{2} g_{0} d_{p} \sqrt{\frac{\Theta}{\pi}} \\
g_{0} & =\frac{1-0.5 \varepsilon_{p}}{\left(1-\varepsilon_{p}\right)^{3}}+\frac{0.58 \varepsilon_{p}^{2}}{\left(\varepsilon_{p}^{\max }-\varepsilon_{p}\right)^{1.5}}
\end{aligned}
$$

Granular temperature equation

$$
\begin{aligned}
& \frac{3}{2}\left[\frac{\partial}{\partial t}\left(\varepsilon_{p} \rho_{p} \Theta\right)+\nabla \cdot\left(\varepsilon_{p} \rho_{p} \mathbf{u}_{p} \Theta\right)\right]=\boldsymbol{\tau}_{p}: \nabla \mathbf{u}_{p}+\nabla \cdot(\kappa \nabla \Theta)-\gamma+\chi \\
& \kappa=2(1+e) \rho_{p} \varepsilon_{p}^{2} g_{0} d_{p} \sqrt{\frac{\Theta}{\pi}}+\varepsilon_{p} \rho_{p} \Theta \frac{\left[1+\frac{3}{5}(1+e)^{2}(2 e-1) \varepsilon_{p} g_{0}\right]\left[1+\frac{6}{5}(1+e) \varepsilon_{p} g_{0}\right]}{\frac{6 K}{5 \rho_{p} \varepsilon_{p}}+\frac{4(1+e)(49-33 e) \varepsilon_{p} g_{0}}{25 d_{p}} \sqrt{\frac{\Theta}{\pi}}} \\
& \gamma=3 K \Theta-\frac{81 \varepsilon_{p} \mu_{g}^{2}\left|\mathbf{u}_{g}-\mathbf{u}_{p}\right|^{2}}{\rho_{p} g_{0} d_{p}^{3} \sqrt{\pi \Theta}} \\
& \chi=-12(1+e)\left(1-e^{\text {eff }}\right) \rho_{p} \varepsilon_{p}^{2} g_{0} \frac{\Theta}{d_{p}} \sqrt{\frac{\Theta}{\pi}}
\end{aligned}
$$


Table S1. (Continued)

Johnson and Jackson boundary conditions

$$
\begin{aligned}
& \mathbf{n} \cdot\left(\boldsymbol{\tau}_{p}+\boldsymbol{\tau}_{f}\right) \cdot \frac{\mathbf{u}_{\text {slip }}}{\left|\mathbf{u}_{\text {slip }}\right|}+\frac{\pi}{2 \sqrt{3} \varepsilon_{p}^{\text {max }}} \phi \rho_{p} \varepsilon_{p} g_{0} \sqrt{\Theta}\left|\mathbf{u}_{\text {slip }}\right|+\left(\mathbf{n} \cdot \boldsymbol{\tau}_{f} \cdot \mathbf{n}\right) \tan \delta_{w}=0 \\
& \mathbf{n} \cdot(\kappa \nabla \Theta)-\frac{\pi}{2 \sqrt{3} \varepsilon_{p}^{\max }} \phi \rho_{p} \varepsilon_{p} g_{0} \sqrt{\Theta}\left|\mathbf{u}_{s l i p}\right|^{2}+\frac{\sqrt{3} \pi}{4 \varepsilon_{p}^{\max }}\left(1+e_{w}\right)\left(1-e_{w}^{\text {eff }}\right) \rho_{p} \varepsilon_{p} g_{0} \Theta \sqrt{\Theta}=0
\end{aligned}
$$


Table S2. Frictional stress model ${ }^{1}$ for particle phase

$$
\begin{aligned}
& \boldsymbol{\tau}_{f}=-p_{f} \mathbf{I}+2 \mu_{f} \mathbf{S}_{p} \\
& \frac{p_{f}}{p_{c}}=\left(1-\frac{\nabla \cdot \mathbf{u}_{p}}{N \sqrt{2} \sin \delta \sqrt{\mathbf{S}_{p}: \mathbf{S}_{p}+\Theta / d_{p}^{2}}}\right)^{\frac{1}{N-1}} \\
& \mu_{f}=\frac{\sqrt{2} \sin \delta p_{f}}{2 \sqrt{\mathbf{S}_{p}: \mathbf{S}_{p}+\Theta / d_{p}^{2}}}\left[N-N(N-1)\left(\frac{p_{f}}{p_{c}}\right)^{\frac{1}{N-1}}\right] \\
& p_{c}=\left\{\begin{array}{cl}
F r \frac{\left(\varepsilon_{p}-\varepsilon_{p}^{\min }\right)^{r}}{\left(\varepsilon_{p}^{\max }-\varepsilon_{p}\right)^{s}} & \varepsilon_{p}>\varepsilon_{p}^{\min } \\
0 & \varepsilon_{p} \leq \varepsilon_{p}^{\min }
\end{array}, \text { with } F r=0.05 \mathrm{~N} / \mathrm{m}^{2}, r=2 \text { and } s=5\right. \\
& N=\left\{\begin{array}{cc}
\frac{\sqrt{3}}{2 \sin \delta} & \nabla \cdot \mathbf{u}_{p} \geq 0 \\
1.03 & \nabla \cdot \mathbf{u}_{p}<0
\end{array}\right.
\end{aligned}
$$


Table S3. Effective restitution coefficient model $^{2}$

$\begin{aligned} e^{\text {eff }}= & e-\frac{1}{2} a_{1}+\frac{1}{2} a_{2} \frac{b_{1}}{b_{2}} \\ a_{1} & =\frac{\mu}{\mu_{0}}\left[\pi \mu_{0}\left(1-\frac{2}{\pi} \arctan \mu_{0}\right)+\frac{2 \mu_{0}^{2}}{1+\mu_{0}^{2}}\left(1-2 \frac{\mu}{\mu_{0}}\right)\right] \\ a_{2} & =\frac{5 \mu}{2 \mu_{0}}\left[\frac{\pi}{2} \mu_{0}\left(1-\frac{2}{\pi} \arctan \mu_{0}\right)+\frac{\mu_{0}^{2}-\mu_{0}^{4}}{1+\mu_{0}^{2}}\right] \\ b_{1} & =\left(\frac{\mu}{\mu_{0}}\right)^{2} \frac{\mu_{0}^{2}}{1+\mu_{0}^{2}} \\ b_{2} & =\frac{\mu}{2 \mu_{0}}\left[\frac{\pi}{2} \mu_{0}\left(1-\frac{2}{\pi} \arctan \mu_{0}\right)+\frac{\mu_{0}^{2}}{1+\mu_{0}^{2}}\right] \\ \mu_{0} & =\frac{7 \mu(1+e)}{2(1+\beta)}\end{aligned}$




\section{NOMENCLATURE}

$\begin{array}{ll}d_{p} & \text { particle diameter } \\ \text { e } & \text { coefficient of normal restitution } \\ \mathbf{g} & \text { gravitational acceleration } \\ g_{\mathbf{0}} & \text { radial distribution function } \\ \mathbf{I} & \text { unit tensor } \\ K & \text { inter-phase momentum transfer coefficient } \\ \mathbf{n} & \text { normal unit vector at the wall } \\ \mathbf{u} & \\ \mathbf{u} & \text { pressure } \\ \mathbf{S} & \\ & \text { strain rate tensor } \\ & \\ & \end{array}$

\section{Greek symbols}

$\begin{array}{ll}\beta & \text { coefficient of tangential restitution } \\ \chi & \text { collisional dissipation of granular energy } \\ \delta & \text { angle of friction for the material } \\ \Delta x, \Delta y, \Delta z & \text { mesh size in the } x, y \text { and } z \text { directions } \\ \varepsilon & \text { volume fraction } \\ \phi & \text { specularity coefficient } \\ \gamma & \text { granular energy exchange between the gas and particle phases } \\ \kappa & \text { conductivity of granular temperature }\end{array}$




$\begin{array}{ll}\mu & \text { Coulomb friction coefficient } \\ \mu_{f} & \text { frictional viscosity of particle phase } \\ \mu_{p} & \text { kinetic-collisional viscosity of particle phase } \\ \Theta & \text { granular temperature } \\ \rho & \text { density } \\ \tau & \text { stress tensor } \\ \tau_{f} & \text { frictional part of particle phase stress tensor } \\ \tau_{p} & \text { kinetic-collisional part of particle phase stress tensor } \\ \xi_{p} & \text { bulk viscosity of particle phase }\end{array}$

\section{Subscripts}

$\begin{array}{ll}g & \text { gas phase } \\ p & \text { particle phase } \\ t & \text { tangential component } \\ w & \text { wall }\end{array}$




\section{REFERENCES}

(1) Srivastava, A.; Sundaresan, S. Analysis of a Frictional-Kinetic Model for Gas-Particle Flow.

Powder Technol. 2003, 129, 72-85

(2) Jenkins, J. T.; Zhang, C. Kinetic Theory for Identical, Frictional, Nearly Elastic Spheres.

Phys. Fluids 2002, 14, 1228-1235. 